\title{
New bisbenzylisoquinoline alkaloid from Laureliopsis philippiana
}

Stærk, Dan; Thi, Loi Pham; Rasmussen, Hasse Bonde; Guzmán, Alfonso; Mølgaard, Per

Published in:

Fitoterapia

DOI:

10.1016/j.fitote.2008.11.002

Publication date:

2009

Document version

Publisher's PDF, also known as Version of record

Citation for published version (APA):

Stærk, D., Thi, L. P., Rasmussen, H. B., Guzmán, A., \& Mølgaard, P. (2009). New bisbenzylisoquinoline alkaloid from Laureliopsis philippiana. Fitoterapia, 80(2), 112-114. https://doi.org/10.1016/j.fitote.2008.11.002 


\title{
New bisbenzylisoquinoline alkaloid from Laureliopsis philippiana
}

\author{
Dan Staerk $^{\mathrm{a}, *}$, Loi Pham Thi ${ }^{\mathrm{b}}$, Hasse Bonde Rasmussen ${ }^{\mathrm{b}}$, Alfonso Guzmán ${ }^{\mathrm{c}}$, Per Mølgaard ${ }^{\mathrm{b}}$ \\ a Department of Basic Sciences and Environment, Faculty of Life Sciences, University of Copenhagen, Thorvaldsensvej 40, DK-1871 Frederiksberg C, Denmark \\ b Department of Medicinal Chemistry, Faculty of Pharmaceutical Sciences, University of Copenhagen, Universitetsparken 2, DK-2100 Copenhagen, Denmark \\ c Universidad de los Lagos, Avenida Fuchslocher 1305, Ozorno, Chile
}

\section{A R T I C L E I N F O}

\section{Article history:}

Received 20 October 2008

Accepted in revised form 11 November 2008

Available online 27 November 2008

\section{Keywords:}

Laureliopsis philippiana

Bisbenzylisoquinoline

Atherospermataceae

NMR

\begin{abstract}
A B S T R A C T
Phytochemical investigation of Laureliopsis philippiana resulted in isolation of a new bisbenzylisoquinoline alkaloid (1) named laureliopsine A. The structure was established by spectroscopic methods, including 2D homo- and heteronuclear NMR experiments. This finding of a bisbenzylisoquinoline alkaloid in Laureliopsis supports its close relationship to Atherosperma and its taxonomic segregation from Laurelia.
\end{abstract}

(c) 2008 Elsevier B.V. All rights reserved.

\section{Introduction}

Laureliopsis philippiana (Looser) Shodde is a slow growing, shade tolerant tree found in Chile and Argentina [1,2]. Extracts of the leaves have traditionally been used for the treatment of colds and headaches by the Mapuche people [3]. Laureliopsis is a monotypic genus belonging to the Atherospermataceae [4], and previous investigations of stem bark constituents revealed the presence of the monomeric aporphinoids asimilobine, anonaine, norcorydine, nornantenine, 4-hydroxynornantenine, and (+)-reticuline [5-7]. In this study, a new 6',7-epoxy analogue (1) of the berbamine subgroup of the bisbenzylisoquinolines was identified in the extract of leaves from Laureliopsis philippiana.

\section{Experimental}

\subsection{General}

Optical rotation was measured using a Perkin-Elmer 241 polarimeter. NMR spectra were recorded on a Bruker Avance NMR spectrometer (proton frequency $600.13 \mathrm{MHz}$ ) at $25{ }^{\circ} \mathrm{C}$, using TMS as internal standard. Gradient selected HMBC and

\footnotetext{
* Corresponding author. Tel.: +45 3533 2425; fax: +45 35332398 .

E-mail address: dst@life.ku.dk (D. Staerk).
}

HSQC spectra were optimized for ${ }^{\mathrm{n}} J_{\mathrm{C}, \mathrm{H}}=7.7 \mathrm{~Hz}$ and ${ }^{1} J_{\mathrm{C}, \mathrm{H}}=145 \mathrm{~Hz}$, respectively, and the NOESY experiment was acquired with a mixing time of $600 \mathrm{~ms}$. High-resolution mass measurements for exact mass determination were performed on a Micromass QTOF spectrometer equipped with an ESI ion source, operating in positive-ion mode. Samples were dissolved in $\mathrm{MeOH}$ and polyethylene glycol (PEG) was added for calibration. Column chromatography was performed on Matrex Silica gel 60A (particle size 70-200 $\mu \mathrm{m}$ ). Alkaloid-containing fractions were monitored using pre-coated silica gel $60 \mathrm{~F}_{254}$ TLC plates using Dragendorff's reagent for visualization.

\subsection{Plant material}

Leaves of Laureliopsis philippiana (Looser) Shodde [Atherospermataceae] were collected in Chile (coastal area close to Pucomo, Ozorno) in October 2006 by Alfonso Guzmán. A voucher specimen (accession number: PM2001/5) has been deposited in Herbarium C (Botanical Museum, University of Copenhagen, Copenhagen, Denmark) as well as at Universidad de los Lagos, Ozorno, Chile.

\subsection{Extraction and isolation}

Powdered leaves (325 g) of L. philippiana were extracted with $\mathrm{CH}_{2} \mathrm{Cl}_{2}-\mathrm{MeOH}$ (1:1) for $6 \mathrm{~h}$ using a Soxhlet extraction 
system. The extract was evaporated under reduced pressure below $40^{\circ} \mathrm{C}$ to yield $67 \mathrm{~g}$ of dry crude extract. The residue was dissolved in $1.5 \mathrm{~L}$ of $0.25 \mathrm{~N} \mathrm{H}_{2} \mathrm{SO}_{4}$ and extracted twice with $1.5 \mathrm{~L}$ of $\mathrm{CHCl}_{3}$. The aqueous layer was basified ( $\mathrm{pH} \mathrm{11)} \mathrm{using}$ $2 \mathrm{~N} \mathrm{NH}_{4} \mathrm{OH}$ and extracted twice with $1.5 \mathrm{~L}_{\text {of }} \mathrm{CHCl}_{3}$. The latter chloroform extract was evaporated as described above to yield $5.95 \mathrm{~g}$ of crude alkaloids. This fraction was subjected to vacuum liquid chromatography (silica gel $60 \mathrm{H}$ (Merck), $8 \times 12 \mathrm{~cm}$ i.d.) and eluted with heptane containing 33-75\% of $\mathrm{CH}_{2} \mathrm{Cl}_{2}-\mathrm{MeOH}$ (9:1). Fractions of $100 \mathrm{~mL}$ were assessed by TLC and visualized with Dragendorff's reagent, and fractions showing similar TLC-profiles were pooled into four fractions A-D. Fraction B (655 mg) was further purified by column chromatography (silica gel 60 ) using a $46 \times 2 \mathrm{~cm}$ i.d. column eluted with $300 \mathrm{~mL}$ of heptane-EtOAc (1:1) followed by $300 \mathrm{~mL}$ of heptane-EtOAc (2:3) to give $24 \mathrm{mg}$ of 1.

Laureliopsine A (1), yellowish gum, $[\alpha]^{25}{ }_{D}=115.6$ (c 0.45 , $\left.\mathrm{CHCl}_{3}\right) ;{ }^{1} \mathrm{H}$ and ${ }^{13} \mathrm{C}$ NMR data: see Table 1 . HR-ESI-TOF-MS $\mathrm{m} / \mathrm{z}$ $575.2500[\mathrm{MH}]^{+}$. Calc. for $\left[\mathrm{C}_{36} \mathrm{H}_{35} \mathrm{~N}_{2} \mathrm{O}_{5}\right]^{+}$575.2541.

\section{Results and discussion}

Repeated chromatographic separation of the leaf extract resulted in isolation of $24 \mathrm{mg}$ of 1 . Combined use of the ${ }^{1} \mathrm{H},{ }^{13} \mathrm{C}$, and DEPT NMR experiments showed the presence of two methoxy groups, two $\mathrm{N}$-methyl groups, five methylenes, 12

Table 1

${ }^{1} \mathrm{H}(600 \mathrm{MHz})$ and ${ }^{13} \mathrm{C}(150 \mathrm{MHz})$ NMR Data of 1

\begin{tabular}{|c|c|c|c|c|c|}
\hline Position & ${ }^{13} \mathrm{C}^{\mathrm{a}}$ & ${ }^{1} \mathrm{H}^{\mathrm{a}, \mathrm{b}}$ & $\operatorname{cosY}^{a, c}$ & NOESY $^{a, c, d}$ & $\mathrm{HMBC}^{\mathrm{a}, \mathrm{c}, \mathrm{e}}$ \\
\hline 1 & 60.3 & $3.62 \mathrm{br} \mathrm{d}\left(J_{\mathrm{H} 1-\mathrm{H} 15 \mathrm{~B}}=10.7\right)$ & H-15A, H-15B & H-8', H-10, H-15A, H-17 & H-3 $\alpha, H-3 \beta, H-5, H-15 A, H-15 B, H-17$ \\
\hline \multirow[t]{2}{*}{3} & 44.1 & $\alpha: 2.82 \mathrm{~m}$ & $H-3 \beta, H-4 \alpha, H-4 \beta$ & $\mathrm{H}-3 \beta, \mathrm{H}-4 \beta, \mathrm{H}-5, \mathrm{H}-17$ & $\mathrm{H}-1, \mathrm{H}-4 \alpha, \mathrm{H}-17$ \\
\hline & & $\beta: 3.40 \mathrm{~m}$ & $H-3 \alpha, H-4 \alpha, H-4 \beta$ & H-3A, H-4ß, H-5, H-15B & \\
\hline \multirow[t]{2}{*}{4} & 22.6 & $\alpha: 2.82 \mathrm{~m}$ & $\mathrm{H}-3 \alpha, \mathrm{H}-3 \beta, \mathrm{H}-4 \beta$ & $\mathrm{H}-4 \beta$ & $\mathrm{H}-3 \alpha, \mathrm{H}-3 \beta, \mathrm{H}-5$ \\
\hline & & $\beta: 2.39 \mathrm{br}$ dd $\left(J_{\mathrm{H} 4 \beta-\mathrm{H} 4 \alpha}=15.9 ; J_{\mathrm{H} 4 \beta-\mathrm{H} 3 \beta}=5.7\right)$ & $\mathrm{H}-3 \alpha, \mathrm{H}-3 \beta, \mathrm{H}-4 \alpha$ & $\mathrm{H}-3 \alpha, \mathrm{H}-3 \beta, \mathrm{H}-4 \alpha$ & \\
\hline $4 a$ & 128.5 & & & & $\mathrm{H}-1, \mathrm{H}-3 \alpha, \mathrm{H}-3 \beta, \mathrm{H}-4 \alpha$ \\
\hline 5 & 106.5 & 6.26 br s & $\mathrm{H}-4 \alpha, \mathrm{H}-4 \beta$ & $\mathrm{H}-4 \alpha, \mathrm{H}-4 \beta, \mathrm{H}-18$ & $\mathrm{H}-3 \alpha, \mathrm{H}-4 \alpha, \mathrm{H}-4 \beta, \mathrm{H}-18$ \\
\hline 6 & 145.9 & & & & $\mathrm{H}-5, \mathrm{H}-18$ \\
\hline 7 & 129.5 & & & & $\mathrm{H}-5$ \\
\hline 8 & 139.6 & & & & $\mathrm{H}-1, \mathrm{H}-5$ \\
\hline $8 \mathrm{a}$ & 119.1 & & & & $\mathrm{H}-1, \mathrm{H}-5, \mathrm{H}-3 \alpha, \mathrm{H}-4 \alpha, \mathrm{H}-15 \mathrm{~A}$ \\
\hline 9 & 134.2 & & & & H-1, H-10, H-13, H-15A, H-15B \\
\hline 10 & 114.6 & $6.64 \mathrm{~d}\left(J_{\mathrm{H} 10-\mathrm{H} 14}=1.9\right)$ & $\mathrm{H}-14$ & H-1, H-11', H-13', H-15A & H-13, H-14, H-15A, H-15B \\
\hline 11 & 149.6 & & & & H-10, H-13, H-15B \\
\hline 12 & 146.6 & & & & H-10, H-13, H-14, H-16 \\
\hline 13 & 111.5 & $6.85 \mathrm{~d}\left(J_{\mathrm{H} 13-\mathrm{H} 14}=8.1\right)$ & $\mathrm{H}-14$ & $\mathrm{H}-14, \mathrm{H}-16$ & $\mathrm{H}-10, \mathrm{H}-16$ \\
\hline 14 & 122.6 & $6.77 \mathrm{~d}\left(J_{\mathrm{H} 14-\mathrm{H} 13}=8.1 ; J_{\mathrm{H} 14-10}=1.9\right)$ & $\mathrm{H}-10, \mathrm{H}-13$ & H-13, H-15B, H-17(weak) & H-10, H-13, H-15A, H-15B \\
\hline \multirow[t]{2}{*}{15} & 41.1 & A: 2.53 br d $\left(J_{H 15 A-H 15 B}=14.0\right)$ & H-1, H-15B & H-1, H-8', H-10, H-15B & H-1, H-4, H-10, H-13 \\
\hline & & B: $2.64 \mathrm{dd}\left(J_{\mathrm{H} 15 \mathrm{~A}-\mathrm{H} 15 \mathrm{~B}}=14.0 ; J_{\mathrm{H} 15 \mathrm{~A}-\mathrm{H} 1}=10.7\right)$ & H-1, H-15A & H-3ß, H-14, H-15A & \\
\hline 16 & 56.1 & $3.96 \mathrm{~s}$ & & $\mathrm{H}-13$ & \\
\hline 17 & 42.8 & $2.22 \mathrm{~s}$ & & $\mathrm{H}-1, \mathrm{H}-3 \alpha, \mathrm{H}-14$ (weak) & $\mathrm{H}-3 \alpha, \mathrm{H}-3 \beta$ \\
\hline 18 & 56.2 & $3.83 \mathrm{~s}$ & & H-5, H-5'(weak) & \\
\hline $1^{\prime}$ & 141.6 & & & & H-3'A, H-3'B, H-8', H-15', H-16' \\
\hline \multirow[t]{2}{*}{$3^{\prime}$} & 50.5 & $\begin{array}{l}\text { A: } 3.16 \text { dt }\left(J_{\mathrm{H} 3^{\prime} \mathrm{A}-\mathrm{H} 3^{\prime} \mathrm{B}}=11.2 ;\right. \\
\left.J_{\mathrm{H} 3^{\prime} \mathrm{A}-\mathrm{H} 4^{\prime} \mathrm{A}}=J_{\mathrm{H} 3^{\prime} \mathrm{A}-\mathrm{H} 4^{\prime} \mathrm{B}}=5.2\right)\end{array}$ & H-3'B, H-4'A, H-4'B & H-3'B, H-4'B, H-16' & H-4'A, H-4'B, H-16' \\
\hline & & $\begin{array}{l}\mathrm{B}: 3.21 \text { ddd }\left(J_{\mathrm{H} 3}{ }^{\prime} \mathrm{B}-\mathrm{H} 3^{\prime} \mathrm{A}\right. \\
\left.J_{\mathrm{H} 3^{\prime} \mathrm{B}-\mathrm{H} 4^{\prime} \mathrm{A}}=4.2\right)\end{array}$ & H-3'A, H-4'A, H-4'B & H-3'A, H-4'A, H-4'B, H-16' & \\
\hline \multirow[t]{2}{*}{$4^{\prime}$} & 30.4 & $\begin{array}{l}\text { A: } 2.76 \text { ddd }\left(J_{\mathrm{H}^{\prime} \mathrm{A}-\mathrm{H} 4^{\prime} \mathrm{B}}=15.4 ; J_{\mathrm{H}^{\prime} \mathrm{A}-\mathrm{H} 3^{\prime} \mathrm{A}}=5.2 ;\right. \\
\left.J_{\mathrm{H} 4^{\prime} \mathrm{A}-\mathrm{H} 3^{\prime} \mathrm{B}}=4.2\right)\end{array}$ & H-3'A, H-3'B, H-4'B & H-3'B, H-4'B, H-5' & H-3'A, H-3'B, H-5', H-16' \\
\hline & & $\begin{array}{l}\mathrm{B}: 2.94 \text { dddd }\left(J_{\mathrm{H}^{\prime} \mathrm{B}-\mathrm{H} 4^{\prime} \mathrm{A}}=15.4 ; J_{\mathrm{H} 4^{\prime} \mathrm{B}-\mathrm{H} 3^{\prime} \mathrm{B}}=9.2 ;\right. \\
\left.J_{\mathrm{H} 4^{\prime} \mathrm{B}-\mathrm{H} 3^{\prime} \mathrm{A}}=5.2 ; J_{\mathrm{H} 4^{\prime} \mathrm{B}-\mathrm{H} 5^{\prime}}=0.8\right)\end{array}$ & H-3'A, H-3'B, H-4'A & H-3'A, H-3'B, H-4'A, H-5' & \\
\hline 4'a & 133.1 & & & & H-3'A, H-3'B, H-4'A, H-4'B, H-5', H-8' \\
\hline $5^{\prime}$ & 115.8 & $6.68 \mathrm{t}\left(J_{\mathrm{H} 5^{\prime}-\mathrm{H} 4^{\prime} \mathrm{A}}=J_{\mathrm{H}^{\prime}-\mathrm{H} 4^{\prime} \mathrm{B}}=0.8\right)$ & H-4'A, H-4'B & H-4'A, H-4'B, H-18 & H-4'A, H-4'B, H-8' \\
\hline $6^{\prime}$ & 140.9 & & & & H-5', H-8' \\
\hline $7^{\prime}$ & 138.9 & & & & H-5', H-8' \\
\hline $8^{\prime}$ & 116.6 & $6.68 \mathrm{~s}$ & & H-1, H-10', H-14', H-15A(weak), & H-5', H-15' \\
\hline 8'a & 126.8 & & & & H-4'A, H-4'B, H-5', H-8', H-15' \\
\hline $9^{\prime}$ & 137.8 & & & & H-11', H-13', H-15' \\
\hline $10^{\prime}$ & 131.2 & $7.37 \mathrm{dd}\left(J_{\mathrm{H} 10^{\prime}-\mathrm{H} 11^{\prime}}=8.1 ; J_{\mathrm{H} 10^{\prime}-\mathrm{H} 14^{\prime}}=2.1\right)$ & H-11', H-15' & H-8', H-11', H-15', H-16' & H-11', H-13', H-14' \\
\hline $11^{\prime}$ & 123.1 & 7.26 m (overlapping with $\left.\mathrm{H}-14^{\prime}\right)$ & $\mathrm{H}-10^{\prime}$ & $\mathrm{H}-10, \mathrm{H}-10$ & $\mathrm{H}-13^{\prime}$ \\
\hline $12^{\prime}$ & 153.0 & & & & H-10', H-11', H-13', H-15' \\
\hline $13^{\prime}$ & 123.7 & $7.09 \mathrm{dd}\left(J_{\mathrm{H} 13^{\prime}-\mathrm{H} 14^{\prime}}=8.4 ; J_{\mathrm{H}_{13}{ }^{\prime}-\mathrm{H} 11^{\prime}}=2.4\right)$ & H-11', H-14' & $\mathrm{H}-10, \mathrm{H}-14$ & $\mathrm{H}-11^{\prime}$ \\
\hline $14^{\prime}$ & 131.2 & 7.26 m (overlapping with H-11') & H-13', H-15' & H-8', H-13', H-15', H-16(weak) & $\mathrm{H}-10^{\prime}$ \\
\hline $15^{\prime}$ & 100.7 & $5.71 \mathrm{br} \mathrm{s}$ & H-10', H-14' & H-10', H-14', H-16' & H-3'A, H-3'B, H-8', H-10', H-14', H-16' \\
\hline $16^{\prime}$ & 41.4 & $2.97 \mathrm{~s}$ & & $\begin{array}{l}\text { H-3'A, H-3'B, H-10'(weak), } \\
\text { H-14'(weak), H-15' }\end{array}$ & H-3'A, H-3'B, H-15' \\
\hline
\end{tabular}

\footnotetext{
${ }^{\text {a }}{ }^{1} \mathrm{H}(600 \mathrm{mHz})$ and ${ }^{13} \mathrm{C}(150 \mathrm{mHz}) \mathrm{NMR}$ spectral data measured in $\mathrm{CDCl}_{3}, \delta$ values relative to internal TMS.

b The multiplicity of signals is given in parentheses: s, singlet; d, doublet; t, triplet; m, multiplet; br, broad. Coupling constants (apparent splittings) are reported as numerical values in $\mathrm{Hz}$.

c Signal correlating with ${ }^{1} \mathrm{H}$ resonance.

d Mixing time $600 \mathrm{~ms}$.

e Optimized for ${ }^{\mathrm{n}} J_{C, H}=7.7 \mathrm{~Hz}$.
} 


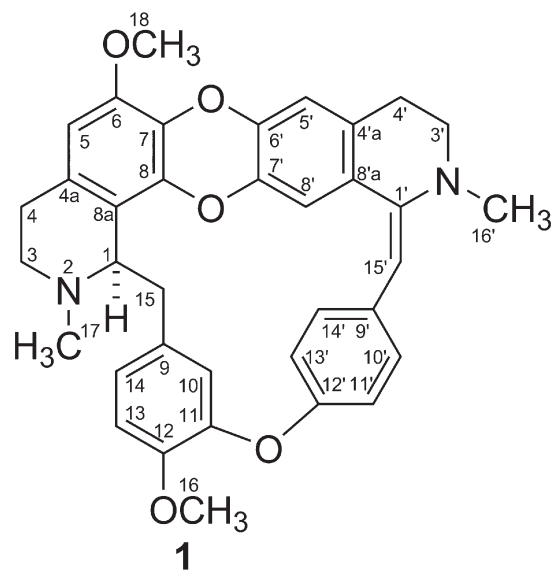

Fig. 1. Structure of laureliopsine A (1).

methines and 15 quaternary carbon atoms, which together with information obtained from MS $\left([\mathrm{M}+\mathrm{H}]^{+} 575.2100\right)$ led to the molecular formula $\mathrm{C}_{36} \mathrm{H}_{34} \mathrm{~N}_{2} \mathrm{O}_{5}$. The structure of 1 (Fig. 1) was established by combined use of COSY, NOESY, HSQC and HMBC experiments. Thus, the spin systems $\mathrm{H}-3-\mathrm{H}-4, \mathrm{H}-1-\mathrm{H}-$ 15 and $\mathrm{H}-10-\mathrm{H}-13-\mathrm{H}-14$ were identified by analysis of the COSY spectrum, and they were correlated with the benzylisoquinoline moiety containing $\mathrm{H}-5$ by correlation pathways found in the NOESY spectrum (Fig. 2). Correlations observed in the NOESY spectrum also established the position of the methoxy groups at C-6 and C-12, respectively, and the $N$ methyl group at position 2. Similarly, analysis of spectral data starting with $\mathrm{H}-15^{\prime}$ as anchoring point allows assignment of all ${ }^{1} \mathrm{H}$ and ${ }^{13} \mathrm{C}$ resonances of the second benzylisoquinoline moiety. Thus, the NOESY correlation pathways $\mathrm{H}-15^{\prime} \rightarrow \mathrm{H}-$ $16^{\prime} \rightarrow \mathrm{H}-3^{\prime} \rightarrow \mathrm{H}-4^{\prime} \rightarrow \mathrm{H}^{\prime} 5^{\prime}$ and $\mathrm{H}-15^{\prime} \rightarrow \mathrm{H}-10^{\prime}$ and $\mathrm{H}-14^{\prime}$ together with correlations found in the HMBC spectrum (see Fig. 2) established the structure of the second benzylisoquinoline moiety, with the unusual exocylic double bond at C-1'. The head-to-head connection of the two moieties was established by NOESY correlations observed between $\mathrm{H}-13$ ' and $\mathrm{H}-10$, between $\mathrm{H}-8$ ' and $\mathrm{H}-15 \mathrm{~A}$ and $\mathrm{H}-1$, and between $\mathrm{H}-5$ ' and $\mathrm{H}-18$ (see Fig. 2). Compound 1 is a new compound for which the name laureliopsine $A$ is suggested.

This is the first report of a bisbenzylisoquinoline alkaloid from Laureliopsis philippiana and this finding is very important from a chemotaxonomic point of view. One of the first alkaloids from this group, berbamine, was isolated from Atherosperma moschatum from Australia more than 100 years ago [8]. The presence of these alkaloids further supports the connection between Laureliopsis and Atherosperma. Together with the genus Nemuaron, these two genera form a well defined clade within the Atherospermataceae, which separated from the genus Laurelia 83 million years ago as estimated in the most recent phylogenetic investigations of the Laurales $[9,10]$. Further investigations of other parts of the plant would be of great interest as well as correlation of future chemotaxonomic information with DNA-based phylogenetic investigations.

\section{Acknowledgements}

NMR equipment used in this work was purchased via grants from "Apotekerfonden af 1991", Copenhagen, and The Danish University of Pharmaceutical Sciences.

\section{References}

[1] Veblen TT, Donoso C, Kitzberger T, Rebertus AJ. Ecology of Southern Chilean and Argentinean Nothofagus Forests. In: Veblen TT, Hill RS, Read J, editors. The Ecology and Biogeography of Nothofagus Forests. Yale University Press; 1996. p. 293.

[2] Lusk CH. Plant Ecol 1999;140:111.

[3] Houghton PJ, Manby J. J Ethnopharmacol 1985;13:89.

[4] Renner SS. Biol Skr 2004;55:441.

[5] Urzúa A, Cassels BK. Tetrahedron Lett 1978;30:2649.

[6] Urzúa A, Cassels BK. Phytochemistry 1982;21:773.

[7] Urzúa A, Cassels BK. Lloydia 1978;41:98.

[8] Bick IRC. Alkaloids from Australian flora. In: Pelletier SW, editor. Alkaloids: Chemical and Biological Perspectives, vol. 10. Oxford: Pergamon; 1996. p. 1.

[9] Renner SS. Am J Bot 1999;86:1301.

[10] Renner SS. Perspect Plant Ecol Evol Syst 1998;1:61.
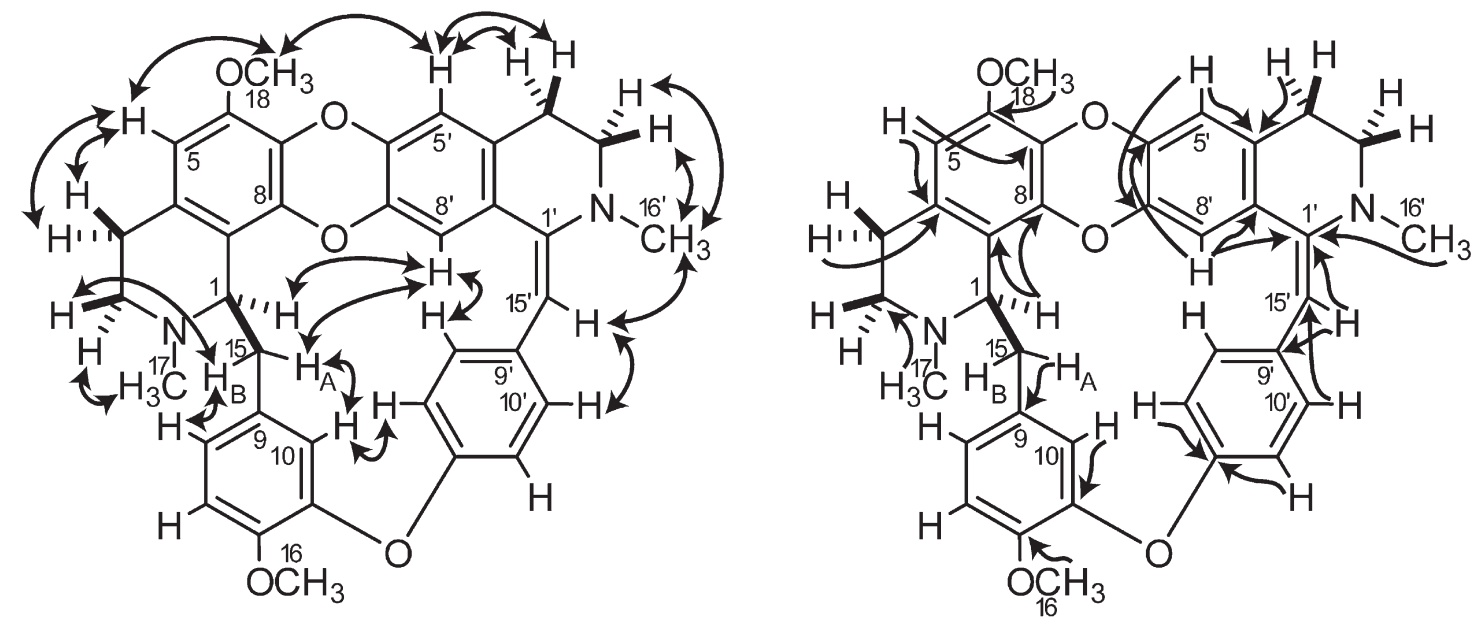

Fig. 2. Selected correlations found in the NOESY experiment (left) and the gradient-selected HMBC experiment (right, arrows indicate correlations from hydrogen to carbon). 